\title{
Some Aspects Concerning the Incidence, Anatomo- Clinical and Profilactico-Therapeutic Picture within Cnemiodocopte Scabie outbreak Pf Legs in Laying Hens
}

\author{
Octavian NEGREA ${ }^{11}$, Camelia RĂDUCU*1), Vioara MIREŞAN ${ }^{1)}$, Mirela CADAR ${ }^{1)}$, Octavia NEGREA ${ }^{2)}$, \\ Aurelia COROIAN ${ }^{1)}$ \\ 1)Faculty of Animal Sciences and Biotechnologies, University of Agricultural Sciences and Veterinary \\ Medicine, 3-5 Mănăștur Street, 400372 Cluj-Napoca, Romania \\ 2)Faculty of Veterinary Medicine, University of Agricultural Sciences and Veterinary Medicine, 3-5 \\ Mănăştur Street, 400372 Cluj-Napoca, Romania \\ *Corresponding author, email: craducu2001@yahoo.com
}

Bulletin UASVM Animal Science and Biotechnologies 71(2) / 2014,

Print ISSN 1843-5262; Electronic ISSN 1843-536X

DOI:10.15835/buasvmcn-asb:10785

\begin{abstract}
The investigation performed on a 31 hens effective (27 laying hens and 4 cocks) reared and maintained in traditional system during May-June 2014, concerning the anatomo-clinical, hystopathological, prophylactic and therapeutic picture in a cnemiodocopte scabie outbreak of legs, emphasizes an incidence of the parasitosis with different values function of production category, $14.8 \%$ in laying hens and $75.5 \%$ in cocks. The anatomo-clinical examination revealed poultry within different stages of disease evolution, from incipient forms, less expressive $(3$ cases-43.0\%) to serious foms, with productive dermatoses high prurigenous in the tarso-metatarso-phalangeal area (4 cases- 57.0\%). The hystopathological sections performed from pathological material, collected from the tarso-metatarsian area with serious cnemiodocopte scabie lesions in hen, emphasize at epidermis level lesions of acanthosis with hyperkeratosis, and spongiosis lesions in the cells of the spinous layer, and around parasites appears a limiting thick layer, keratinic. In deep dermis is presented an infiltrate preponderantly perivascular, with mononucleates and hetherophils (lesions of perivascularite). According to applied therapeutic protocol (Ivermectin, $0.5 \mathrm{ml}$ s.c. inoculation in axilar area) it was reported a progressive improvement of the anatomoclinical picture, visible from the $7^{\text {th }}$ post-therapeutic day.
\end{abstract}

Keywords: scabie, prurit, crust, hyperkeratosis

\section{INTRODUCTION}

The cnemiodocopte scabie is part of serious arachnoses affecting numerous domestic and wild poultry species. The presence of the disease in poultry effectives, in laying hens, especially, appears on the body or at legs level and determines anatomo-clinical disorders as crust prurigenous dermatitis, plum disappearance or hyperkeratosis with legs deformation. The poultry exhibit a craving state, with decreasing of the feeding time. These produce important economic damages, by decreasing the egg production and carcass depreciation. From these considerations in this paper we aim to an epizootic, anatomo-clinical, and prophylactic and therapeutic study, within a cnemiodocopte scabie outbreak of legs, in laying hens from a traditional house holding located in rural area.

\section{MATERIALS AND METHODS}

The research concerning the cnemiodocopte scabie incidence, anatomo-clinical picture and applied therapeutic protocol were performed during May-June 2014, on an effective of 31 
Tab. 1. The incidence cnemiodocopte scabie of legs in poultry (hens and cocks)

\begin{tabular}{cccc}
\hline \multirow{2}{*}{ Category } & \multirow{2}{*}{ Poultry effective } & \multicolumn{3}{c}{ of which } \\
\cline { 3 - 4 } & 27 & positive & $\%$ \\
\hline Laying hens & 4 & 4 & 14.8 \\
\hline Cocks & 31 & 3 & 75.0 \\
\hline Total & 7 & & 22.5 \\
\hline
\end{tabular}

poultry (27 laying hens and 4 cocks), from a rural house holding.

The studied poultry effective was made-up of laying hens, common breed, aged of 1-2 years, reared and maintained within household place and foraging based on whole concentrates (maize, wheat) and access to grass. An anatomo-clinical examination was performed by inspection, in order to identify the affected individuals (congestive lesions, prurit, unaesthetic aspect, crust). From leg scabie suspected poultry (7 cases) prelevations of pathological material were performed, individually, by scraping with a scalpel in sterile tubes. From these, microscopic preparations were obtained, in order to formulate the diagnosis.

From positive diagnosed poultry by microscopic examination, a single hen was scarified and members with crust lesions were prelevated. From these members, hystological preparations were obtained, hematoxylin-eosin (HE) stained. The positive diagnosed poultry were treated with avermectine, by $0.5 \mathrm{~mL}$ Ivermectin subcutaneous inoculation in axilar zone, notifying the disease evolution, and evaluating the anatomo-clinical picture after 7 days and 14 days.

\section{RESULTS AND DISCUSSION}

1. The values resulted from the microscopic examination of preparations obtained from crust harvested from poultry suspected of disease, concerning the incidence of cnemiodocopte scabie of legs by present poultry effective, are presented in Table 1.

From obtained data concerning the incidence of the cnemiodocopte scabie of legs in poultry, function of production category results percentage values with large differences between the both categories: $14.8 \%$ laying hens and $75.0 \%$ cocks.

The parasitosis has an occasionally character rarely appears in hens maintained in batteries, opposed to those reared on permanent litter. Although disease diffusivity even reduced, is carried-out through a direct contact sick birdhealthy bird. As a result of contamination, parasites develop both at skin surface and within epidermal layers reaching the level of the basal

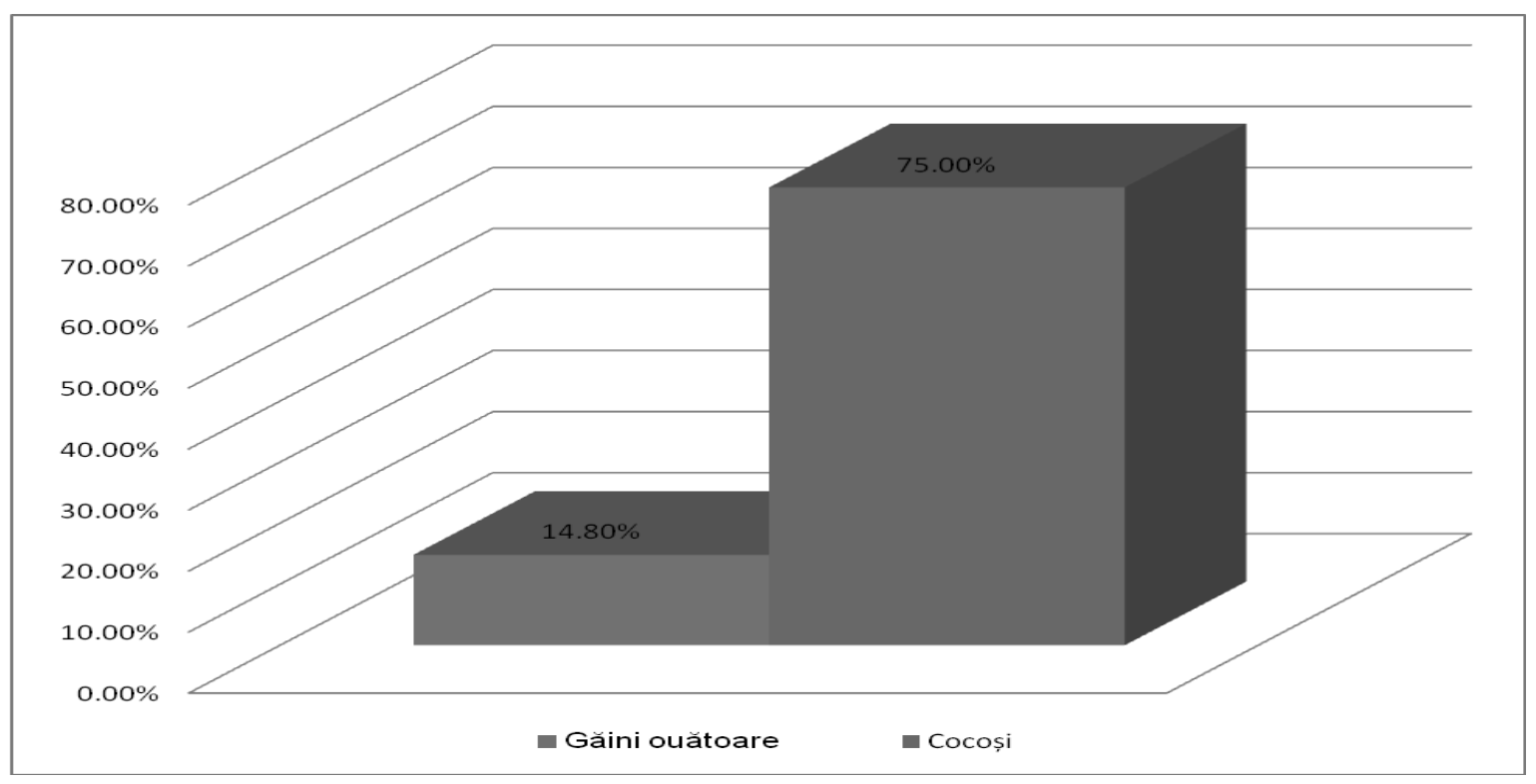

Fig. 1. The variation of the cnemiodocopte scabie of legs extent in poultry by age categories 
membrane, in tarso-metatarso-pharingeal regions. It is noticeable that under formed crust, of specific aspect, in females there are formed real lodges with micro-cannons. In the following figure (Fig. 1 ) is presented the variation of cnemiodocopte

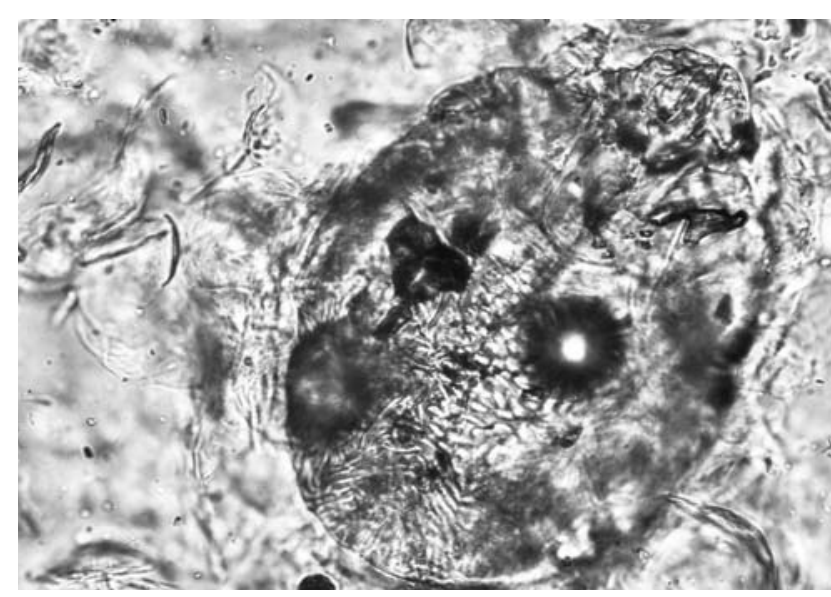

Fig. 2. Cnemidocoptes mutans, femele, objective 40x (original photo)

scabie of legs extent, in poultry by age categories.

The microscopic preparates performed from crust scraping with lacto-phenol supplements emphasize, within microscopic field, the parasites identified in different evolutive stages (Fig. 2).

2 . The results of the anatomo-clinical examinations obtained by macroscopic inspection, performed within gallinaceans effective, are presented in Table 2.

The value of the anatomo-pathological examination obtained as consequence of an attentive inspection poultry affected by the cnemiodocopte scabie of legs emphasizes the presence of a picture of the clinical and lesional manifestations characteristic to scabie outbreaks, whatever affected specie. The symptomatic tetrad (prurit, unaesthetic aspect, crust forming, plum disappearance) also appears in studied group of hens affected by cnemiodocopte scabie of legs, but with small differences. We have to mention that, scabie, as pathological state, evolves during a long time-period (months), interval in which the anatomic -clinical picture is progressively contoured, becoming characteristic, almost pathognomic (specific) the thickening of limbs. The anatomo-clinical examination emphasizes poultry with different stages of evolution, from incipient forms, less expressive (3 cases, $43 \%$ ) to serious forms, with spongious crusts, thick, white, deformed (4 cases $57 \%$ ).

Poultry are hardly moving, remain in prolonged decubitus, do not eat, so start to loose weight. The presence of parasites in large numbers in deep epidermal structures, with active movement favors the emergence of a pronounced itching that oblige bird to peck continuously and this is leading to self-mutilation, in particular cocks. Also, the egg production drops (Kato, 1972; Suteu and Cozma,

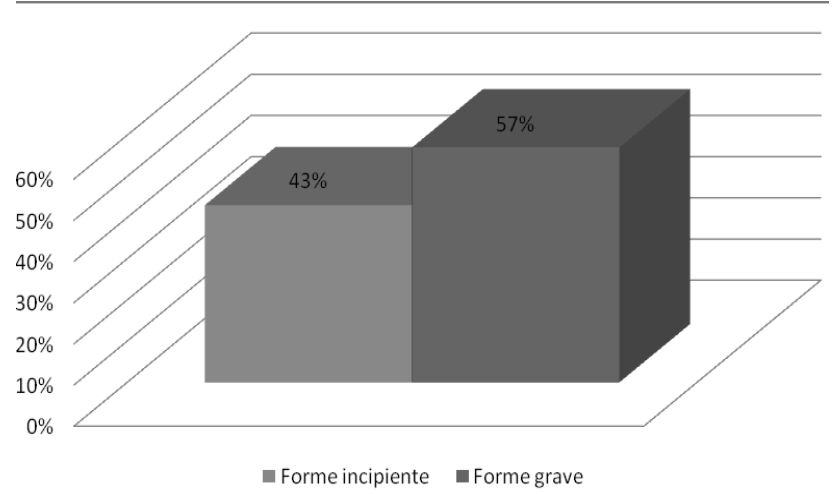

Fig. 3. The percent of poultry with different stages of evolution of cnemiodocopte scabie

1998).

In joined Figure 3 is presented the variation of the anatomo-clinical picture of the cnemiodocopte scabie of limbs, in studied bird group, in correlation to time-period.

Tab. 2. The results of the anatomo-pathological examination performed on laying hens with cnemiodocopte scabie

\begin{tabular}{ccccc}
\hline $\begin{array}{c}\text { No. } \\
\text { sample }\end{array}$ & Prurit & $\begin{array}{c}\text { Anatomo-clinical examination } \\
\text { aspect }\end{array}$ & Crust forming & $\begin{array}{c}\text { Thickening and } \\
\text { deformation of } \\
\text { limbs }\end{array}$ \\
\hline 1 & + & + & + & - \\
\hline 2 & + & + & - & - \\
\hline 3 & + & + & + & + \\
\hline 4 & + & + & + & + \\
\hline 5 & + & + & + & + \\
\hline 6 & + & + & - & + \\
\hline 7 & + & + & + & + \\
\hline
\end{tabular}




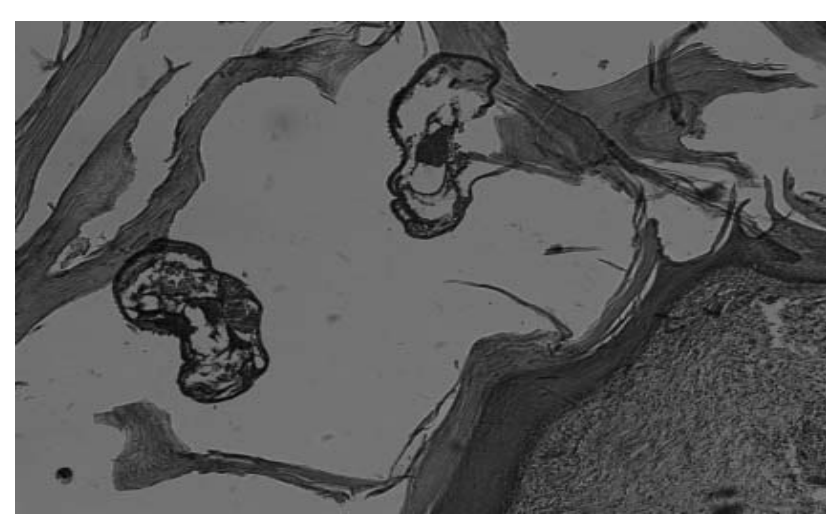

Fig. 4. The hystopathological section of cutaneous tissue from limbs (ob.4x and 10x ) HE stained (original photo)

3. The hysto-pathological sections performed on pathological material prelevated from metatarso-phalangeal area prelevated from a hen affected by emphasize an anatomo-pathologic picture characteristic to this parasitosis (Fig. 4.).

The histopathological sections made from collected pathological material reveals lesions of acanthosis with orthokeratotic hyperkeratosis in the epidermis, keratin having lamellar appearance and hypertrophied. In the mean time, in epidermis appears sponginess lesions mainly in the spinous layer cells and around the parasite emerges a thick, keratin, boundary layer (Avian Histopathology, 1983). At the level of the dermo-epidermal junction is present a discrete multifocal infiltration with mononuclear and heterophil cells. In the mean time, the cells from the basal layer are flattened latero-lateral and exhibit intercellular edema. At the level of deep dermis appears a perivascular infiltrate with mononuclear and heterophyle cells (lesions of perivascularite).

4. Treatement. As consequence of the therapeutic protocol applied (subcutaneous inoculation of $0.5 \mathrm{~mL}$ in axilar region) a progressive improvement was reported for the anatomo-clinical picture, visible beginning with the $7^{\text {th }}$ post-therapeutic day, expressed by:

- reducing of itching and pecking

- return of appetite

- reduction of crust and desquamation

- decrease of locomotion disorders

- return of laying.

\section{CONCLUSION}

The investigation performed on a 31 hens effective (27 laying hens and 4 cocks) reared and maintained in traditional system during May-June
2014, concerning the incidence, anatomo-clinical, hystopathological, prophylactic and therapeutic picture in a cnemiodocopte scabie outbreak of limbs, emphasizes the following aspects:

The incidence of cnemiodocopte scabie of limbs, function of production category, emphasizes very different values between both categoriies, $14.8 \%$ in laying hens and $75.5 \%$ in cocks.

The anatomo-clinical examination revealed poultry within different stages of disease evolution, from incipient forms, less expressive (3 cases-43.0\%) to serious forms, with productive dermatoses high prurigenous in the tarso metatarso - phalangeal area (4 cases - 57.0\%).

Thehystopathologicalsectionsperformedfrom pathological material prelevated from the tarso - metatarsian area with serious cnemiodocopte scabie lesions in hen, emphasize at epiderm level lesions of acanthosis with hyperkeratosis, and spongiosis lesions in the cells of the spinous layer, and around parasites appears a limiting thick layer, keratinic. In deep dermis is presented an infiltrate preponderantly perivascular, with mononucleates and hetherophils (lesions of perivascularite).

According to applied therapeutic protocole (Ivermectin, $0.5 \mathrm{ml}$ s.c. inoculation in axilar area) it was reported a progresive improvement of the anatomo-clinical picture, visible from the $7^{\text {th }}$ posttherapeutic day.

\section{REFERENCES}

1. AVIAN HISTOPATHOLOGY (FirstEdition) (1983). Published by The American Association of Avian Pathologists.

2. Bala AY, Anca SA, Waziri A, Shehu H (2011). Preliminary survey Of Ectoparasites Infesting Chikens in four areas of Sokoto Metropolis, Department of Biological Sciences Usmanu Danfodiyo University, Sokoto, 173-177.

3. Cosoroabă I (2000). Parazitologie Veterinară, Ed. Mirton, Timişoara.

4. Cozma V, Negrea O, Gherman C (2007). Diagnosticul bolilor parazitare la animale. Ed. Genesis, Cluj-Napoca

5. Mehlhorn H (2001). Mites. Integument. In: Encyclopedic Reference of Parasitology. Biology. Structure.

6. Procter H, I Owens (2000). Mites and birds: diversity, parasitism and coevolution, Trends. Ecol. Evol.

7. Ryant $T$ (1987). Cnemidocoptic mite infestation in cage birds.Clinical Insight.

8. Saif YM (2008). Diseases of poultry, Blackwell Publishing, Iowa, 1016-1020.

9. Saif YM (2008). Diseases of poultry, Blackwell Publishing, Iowa, 1011-1013.

10. Şuteu I, V Cozma (2007). Parazitologie Clinică Veterinară, Vol I,II. Ed. Risoprind, Cluj-Napoca. 